\title{
Near Wall Dynamics of Premixed Flames
}

\author{
Feichi Zhang ${ }^{\mathrm{a}, *}$, Thorsten Zirwes ${ }^{\mathrm{b}, \mathrm{a}}$, Thomas Häber ${ }^{\mathrm{c}}$, Henning Bockhorn ${ }^{\mathrm{a}}$, Dimosthenis Trimis ${ }^{\mathrm{a}}$, Rainer Suntz ${ }^{\mathrm{c}}$ \\ ${ }^{a}$ Engler-Bunte-Institute, Division of Combustion Technology, Karlsruhe Institute of Technology, Engler-Bunte-Ring 1, 76131 Karlsruhe, Germany \\ ${ }^{b}$ Steinbuch Centre for Computing (SCC), SimLab Energy $\mathcal{G}$ Competence Centre ING, Karlsruhe Institute of Technology, \\ Hermann-von-Helmholtz-Platz 1, Karlsruhe, Germany \\ ${ }^{c}$ Institute of Chemical Technology and Polymer Chemistry, Karlsruhe Institute of Technology, Engesserstr. 20, 76131 Karlsruhe, Germany
}

\begin{abstract}
Highly-resolved numerical simulations employing detailed reaction kinetics and molecular transport have been applied to flame-wall interaction (FWI) of laminar premixed flames. A multiple plane-jet flame (2D) has been considered, which is operated with premixed methane/air mixtures at atmospheric conditions and with different equivalence ratios. Free flame (FF) and side-wall quenching (SWQ) conditions have been accomplished by defining one lateral boundary as either a symmetry plane for FF or a cold wall with fixed temperature for SWQ. An equidistant grid with a resolution of $20 \mu \mathrm{m}$ is used to resolve the FWI zone. The GRI-3.0 mechanism is used for computing chemical reaction rates. The flame is tangentially compressed when approaching the cold wall, and elongated in the FF case, causing an inversion of the sign of the tangential strain rate $K a_{s}$ and a considerable decrease of the total stretch rate $K a_{t o t}$ for the SWQ flame. The flame consumption speed $S_{L}$ decreases with decreasing normal stretch due to curvature $K a_{c}$ while approaching the cold wall, but it increases with decreasing $K a_{c}$ for the FF case, leading to an inversion of the Markstein number $M a_{t o t}$ based on $K a_{t o t}$ from positive in FF to negative in the SWQ case. The results reveal a strong correlation of flame dynamics during transitions from FWI to freely propagating flames, which may bring a new perspective for modeling FWI phenomena by means of flame dynamics. To do this, the quenching effect of the wall may be reproduced by an inversion of the Markstein number from positive to negative in the FWI zone and applying the general linear Markstein correlation, leading to a decrease of the flame consumption speed. In addition, the quenching distance evaluated from $S_{L}$ has been found to be almost equal to the unstretched laminar flame thickness, which compares quantitatively well with measured data from literature.
\end{abstract}

Keywords: Side wall quenching; Flame Dynamics; Flame wall interaction; Direct numerical simulation; OpenFOAM;

${ }^{*}$ Feichi Zhang

Email address: Feichi.Zhang@kit.edu (Feichi Zhang) 


\section{Introduction}

\subsection{Flame-Wall Interaction (FWI)}

Most engineering applications of combustion processes with high power densities, for instance in gas turbines, internal combustion engines or aero engines, take place in an enclosed chamber, where the flames propagate in the vicinity of walls and interact with them. The wall heat fluxes significantly impact the efficiency and stability of the flame, but also the pollutant formation and lifetime of combustion chambers [1]. Extensive experimental and numerical studies on FWI have been made in recent years [2]-10], which improved the understanding of the phenomena substantially. For instance, Dreizler and Böhm [2] performed comprehensive laser diagnostics for a detailed measurement of premixed methane/air flames in the FWI zone, including gas phase flow velocity, temperature, concentrations of chemical species and flame structures. The measured data from [2] have then been successfully reproduced by Ganter et al. [5] and Heinrich et al. [4] by performing numerical simulations. Häber and Suntz [6] and Strassacker et al. [7] showed experimental and numerical studies regarding the effect of different wall materials on FWI. Mejia et al. [8] studied the influence of wall temperatures on the flame response to acoustic perturbations. The work by Kosaka et al. [9] focused on the $\mathrm{CO}$ formation/oxidation of premixed methane/air and DME/air flames for a side-wall quenching case. The numerical simulation by Poinsot et al. [10] showed that the wall can affect the flame evolution even if the distance between the flame and the wall is significant. Popp and Baum [11] presented a numerical study of FWI for a stoichiometric methane/air flame, where a one-step chemical reaction fails to predict heat fluxes through the wall.

From the numerical point of view, the reaction model needs to take the heat loss due to an extremely high temperature gradient in the FWI zone into account. This is commonly accomplished by using an additional control parameter, i.e., the enthalpy loss in the framework of the flamelet approach; otherwise, reaction models using Arrhenius-type equations for the reaction rates have to be used [1, 4, 5, 12, 13]. The flamelet concept requires generating chemistry tables with 1D canonical flames with lowered system enthalpies, for example, in terms of reduced unburnt temperatures. The Arrhenius-type reaction rates are computationally very expensive when using complex reaction mechanisms, which is however mandatory for FWI computations in order to include low-temperature kinetics and to correctly capture the wall heat fluxes [1]. Previous numerical works on FWI were performed mostly with under-resolved grid resolutions or for a small flame branch to save computing time [4, 5, 7, 14]. Focus of these works was also to validate the proposed modeling concepts or phenomenological effects such as the influences of using detailed reaction mechanisms and transport, or wall materials. Moreover, flames approaching walls are dominated by unsteady effects, which further complicate the phenomena and makes the comparison between measurement and simulation difficult. In summary, despite the technological advances in laser diagnostics and high performance computing, studying FWI is challenging, as the relevant phenomena occur in a very thin near-wall layer with a thickness of the order of $O(0.1) \mathrm{mm}$. Therefore, more fine-grained simulations of FWI with sufficiently high grid resolutions, detailed reaction and transport models, as well as well-defined boundary conditions are necessary for a thorough understanding of the underlying processes of FWI.

\subsection{Flame Dynamics}

Flame speed represents one of the most fundamental parameters for the characterization of combustion processes, which, for example, can be used to predict flame stability or flame length for designing burner systems. It can also be used to model the burning rate of fuel or the intrinsic propagation speed of the flame surface for combustion simulations. The flame speed depends not only on the thermo-physical properties of the ignitable mixture but also on hydrodynamic properties such as stretch caused by a non-uniform flow [1]. These flow inhomogeneities modify the internal flame structure by varying the local balance between chemical reactions and transport (convection-diffusion) inside the flame. The correlation between flame speed and stretch is helpful for describing phenomena such as flame propagation, stabilization or extinction through stretch-induced change of the flame layers.

Flame stretch is defined as the logarithmic Lagrangian time derivative of the area $A$ of an element on the flame surface, which can be calculated from [1, 15]:

$$
K \equiv \frac{1}{A} \frac{\mathrm{d} A}{\mathrm{~d} t}=(\mathbf{I}-\vec{n} \vec{n}): \nabla \vec{u}+S_{d} \nabla \cdot \vec{n}
$$

where $\mathbf{I}$ is the unit tensor, $\vec{u}$ the flow velocity and $\vec{n}$ the unit normal vector. The first term $K_{s}=(\mathbf{I}-\vec{n} \vec{n}): \nabla \vec{u}$ on the r.h.s. in Eq. (1) is attributed to hydrodynamic stretch caused by the flow and the second term $K_{c}=S_{d} \nabla \cdot \vec{n}$ due to the motion of a curved surface with the displacement speed $S_{d}$ along the flame normal direction. $\vec{n}=\frac{\nabla Y_{F}}{\left|\nabla Y_{F}\right|}$ 
and $S_{d}$ are evaluated at the flame surface defined by the fuel mass fraction $Y_{F}=Y_{F \text {,iso }[16]}$

$$
S_{d}=-\frac{1}{\left|\nabla Y_{F}\right|} \frac{\mathrm{d} Y_{F}}{\mathrm{~d} t}=-\frac{\dot{r}_{F}+\nabla \cdot\left(\rho D_{F} \nabla Y_{F}\right)}{\rho\left|\nabla Y_{F}\right|}
$$

$\dot{r}_{F}$ is the reaction rate of fuel, $\rho$ the density and $D_{F}$ the diffusion coefficient of fuel.

The laminar flame speed can be expressed as the consumption speed of the fuel, which is evaluated in the present work by integration of the fuel reaction rate along its local normals

$$
S_{L}=\frac{-1}{\rho_{u}\left(Y_{F, u}-Y_{F, b}\right)} \int \dot{r}_{F} \mathrm{~d} \vec{n}
$$

where $Y_{F, u}$ and $Y_{F, b}$ are the fuel mass fractions in the unburnt and burnt mixtures. Unlike $S_{d}, S_{L}$ represents an integral value that is not dependent on the choice of iso-surface defining the flame front.

The stretched laminar flame speed can be written by a first order Taylor approximation [15] as:

$$
S_{L} / S_{L}^{0}=1-M a \cdot K a
$$

where $K a=K \delta_{L}^{0} / S_{L}^{0}$ represents a normalized stretch rate with the unstretched laminar flame speed $S_{L}^{0}$ and the thermal flame thickness $\delta_{L}^{0}$ defined with the ratio of the temperature difference in burnt and unburnt state to the maximum temperature gradient [1]. The Markstein number $M a$ measures the rate of change in flame speed due to stretch. A positive $M a$ indicates a decrease of $S_{L}$ with $K a$ and vice versa for negative $K a$.

The effect of stretch on flame speed has been extensively studied in the last decades [16-22] and the studies justify the asymptotic correlation given in Eq. (4) for low stretch values. Most of the previous studies have been conducted for 1D outwardly expanding spherical flames and counterflow flames. The spherical flame is only positively curved and the opposed counterflow flame is only positively strained in tangential direction. For a realistic flame, however, flame dynamics are determined by both curvature and tangential strain together. In addition, the influence of heat loss on flame dynamics, for instance, due to a cold wall cannot be assessed. Therefore, detailed studies on flame dynamics are needed to extend existing knowledge for the combined impact of strain and curvature considering the negative stretch range, but also the effect of heat loss.

In order to overcome the limitations in terms of FWI and flame dynamics discussed in the last sections and to study correlations between both effects, direct numerical simulations (DNS) have been performed in this work for a side-wall quenching (SWQ) setup with a 2D laminar plane-jet flame operated with premixed methane/air mixtures. Detailed chemical kinetics and molecular transport models are used and the FWI zone is resolved with a sufficiently fine grid. Special focus of the work is put on the analysis of local flame dynamics regarding flame stretch, flame speed and Markstein effects in the FWI zone. The total length and width of the computational domain are deliberately kept small in order to span a large range of stretch, including both positive and negative values. The work reveals systematically the significant role of flame dynamics for elucidating the mechanism of flame extinguishing at a cold wall.

\section{Numerical Setups}

The 2D computational domain has a rectangular shape with a length of $32 \mathrm{~mm}$ in streamwise and $8 \mathrm{~mm}$ in spanwise direction, as illustrated in Fig. 1 Fresh gas with premixed methane/air mixture enters the domain in the positive $y$-direction at the inlet with a temperature of $T_{0}=293 \mathrm{~K}$ and pressure of $p_{0}=1 \mathrm{bar}$. The burnt gas leaves the domain at the outlet, where the gradients of velocity, temperature and all chemical scalars are set to zero. The partially non-reflecting boundary condition proposed by Poinsot and Lelef [23] has been applied to the inlet and outlet boundaries for pressure to avoid reflections of acoustic waves at these boundaries. A parabolic velocity profile given by the Poiseuille solution for laminar pipe flow is used at the inlet with a minimum velocity of zero in the center and a maximum value of $\hat{u}=1.6 \mathrm{~m} / \mathrm{s}$ at both lateral boundaries. This leads to a V-shaped flame stabilized at the center near to the inlet plane, with two half-

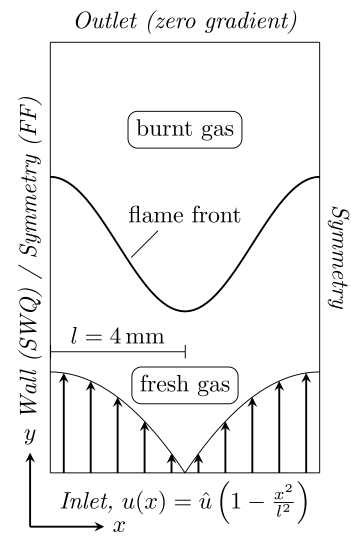
flames on both lateral sides. Figure 1: Schematical drawing Two sets of simulations have of the computational domain. been carried out, with the left boundary defined as a cold wall with $293 \mathrm{~K}$ and as a symmetry plane. The right boundary of the domain is set as symmetry for both cases. These two simulation setups are indentified in the following work with the abbreviations SWQ (side-wall quenching) and FF (free flame), meaning that the left part of flame branch propagates against a cold wall or a symmetry plane.

Premixed methane/air flames at different equivalence ratios $\Phi$ are examined in this work. Table 1 lists flame parameters obtained from detailed computations of 1D 
unstretched freely propagating flames. The flame surface is identified by the mass fraction of fuel corresponding to the highest consumption rate of fuel $Y_{\dot{r}_{\max }}$ in the unstretched flame. The chemical reactions of the system are described by the GRI-3.0 mechanism [24] and molecular diffusion is considered with the mixtureaveraged transport model. An equidistant grid length of $20 \mu \mathrm{m}$ is used, which resolves the unstretched laminar flame thickness with more than 20 cells. The simulations have been run with a time step of $0.25 \mu \mathrm{s}$, allowing a maximum CFL number of 0.2 .

Table 1: Flame parameters from 1D flame calculations at different $\Phi$

\begin{tabular}{c|ccc}
$\phi[-]$ & 0.8 & 1.0 & 1.2 \\
\hline$S_{L}^{0}[\mathrm{~cm} / \mathrm{s}]$ & 26.17 & 36.41 & 32.19 \\
$\delta_{L}^{0}[\mathrm{~mm}]$ & 0.536 & 0.445 & 0.472 \\
$Y_{\dot{r}_{\text {max }}}[-]$ & 0.0039 & 0.0057 & 0.0068
\end{tabular}

A DNS solver developed in the framework of OpenFOAM has been used to solve the governing equations in their compressible formulations. The detailed calculations of reaction rates and transport coefficients have been done with optimized algorithms provided by Cantera [25]. The simulations employ a fully implicit scheme of second order (backward) for the time derivative and a fourth order interpolation scheme for the discretization of the convective term. All diffusive terms are discretized with an unbounded scheme of fourthorder, too. The pressure-implicit split-operator (PISO) algorithm has been used for pressure correction. The DNS capability of OpenFOAM has been validated extensively by different authors [16, 26-30], both for nonreactive and reactive flows as well as for different flame configurations, where the simulations made with OpenFOAM showed quantitatively good agreements with results obtained from measurements and other DNS codes. A detailed description of the used DNS solver can be found in [25, 28, 31, 32].

\section{Results and Discussions}

\subsection{Flame Stretch}

Figure 2 on the left displays contours of calculated temperature $T$ for the SWQ case with $\Phi=1$, where the flame surface is given by the solid line as the iso-surface of $Y_{\mathrm{CH}_{4}}=Y_{\dot{r}_{\max }}$ (see Tab.1). As the flame is influenced by the cold wall and the reaction rate is close to zero in the near-wall region, the above definition of flame surface is only valid in the FF range. The fuel concentration decreases along the wall by passing through the FWI zone due to species diffusion. The left part of the
V-shaped flame interacts with the cold wall, where $T$ drastically decreases in the FWI zone. The center plot in Fig 2 shows contours of local consumption rate of fuel $\dot{r}_{\mathrm{CH}_{4}}$, along with the computational grid. The iso-surface $Y_{\mathrm{CH}_{4}}=Y_{\dot{r}_{\max }}$ is given by the white solid curve and the black solid lines indicate flame surface normals at different distances to the wall (numbered from 1 to 4 ). The profiles of $\dot{r}_{\mathrm{CH}_{4}}$ along these flame surface normals are shown on the right of Fig 2, with the blue curve given by the solution of a 1D unstretched flame from Cantera. The flame front is resolved by approx. 20 grid points in the near-wall region, validating the used grid resolution. The profiles of $\dot{r}_{\mathrm{CH}_{4}}$ for lines 1 to 4 are shown against the flame normal coordinate $s / \delta_{L}^{0}$ and are shifted, so that their peak values coincide with each other. The calculated profiles with DNS differ from the Cantera solution due to the influence of stretch and heat loss near the cold wall. In addition, adaptive mesh is used by Cantera, resolving the flame with a finer grid. Although not shown here, similar behaviors are obtained for the other cases with different $\Phi$.

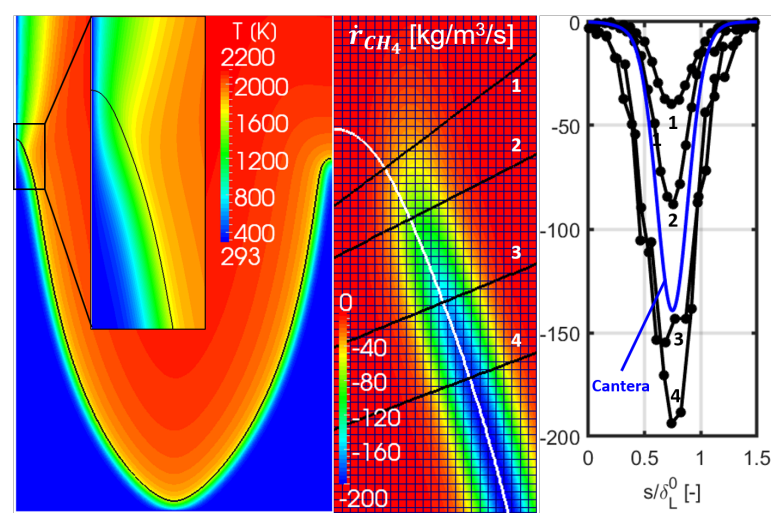

Figure 2: Contour-plots of temperature (left) and consumption rate of fuel $\dot{r}_{\mathrm{CH}_{4}}$ (center), as well as profiles of $\dot{r}_{\mathrm{CH}_{4}}$ along flame normal axis for the SWQ case with $\Phi=1$.

Figure 3 presents calculated $K a_{s}$ (left) and $K a_{c}$ (right) according to tangential and normal stretch (see Eq.(1)) along the lateral axis $x$, with $x=0$ denoting the location of the wall in case of SWQ. Outside the flame tip region with $x / \delta_{L}^{0}>1, K a_{s}$ and $K a_{c}$ are similar for SWQ and FF cases with different $\Phi$, where the flame is only weakly stretched with $K a_{c} \approx 0$. In the near-wall region with $x / \delta_{L}^{0}<1, K a_{s}$ reverses its sign from positive to negative in SWQ compared to FF case. The flame experiences a compression in the SWQ case instead of an elongation in case of FF. The mechanism is explained in Fig 3 on the right by the contours and the streamlines of the flow velocity, where the flame fronts are shown by 
iso-contours of $\dot{q}$. In case of FF, the flow is accelerated by passing through the flame tip, whereas it is decelerated in the SWQ case due to stagnation points generated by wall. The flame curvature decreases rapidly at the tip of the flame leading to large negative values of $K a_{c}$, as shown in Fig 3 on the right. $K a_{c}$ remains negative sign when a cold wall is placed as boundary instead of a symmetry plane. The range of $K a_{c} \approx 0$ extends further towards the wall, resulting in a faster decline of $K a_{c, S W Q}$ compared with $K a_{c, F F}$.
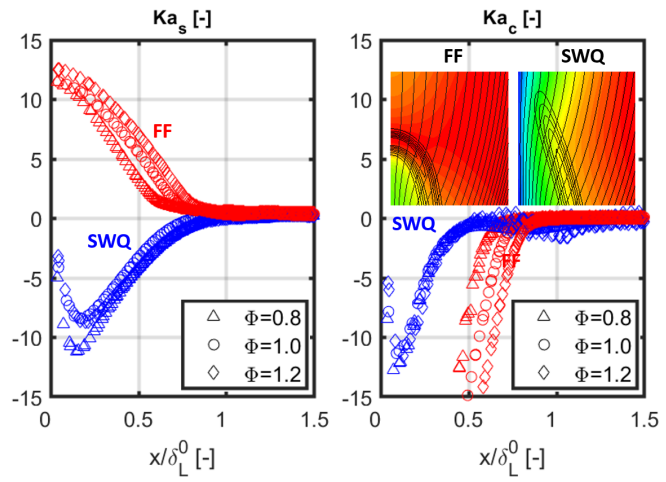

Figure 3: Comparison of $K a_{s}$ (left) and $K a_{c}$ (right) along the wall normal coordinate under SWQ (blue) and FF (red) conditions.

The joint effect of $K a_{s}$ and $K a_{c}$ is shown in Fig 4 on the left by $K a_{\text {tot }}=K a_{s}+K a_{c}$ over $x / \delta_{L}^{0}$, where $K a_{t o t, S W Q}$ starts earlier to decrease while approaching $x=0$ compared with $K a_{t o t, F F}$. A reduction of $K a_{s}, K a_{c}$ and $K a_{t o t}$ with decreasing wall distance was also reported in experiments by [33] for a head-on quenching configuration with premixed methane/air flames. Figure 4 on the right shows the normalized flame consumption speed $S_{L}$ evaluated with Eq. (3) over the wall normal distance. $S_{L}$ is largest at $x / \delta_{L}^{0}=0$ in case of FF and lowest with $S_{L}=0$ for the SWQ case, indicating a completely reversed response of $S_{L}$ to flame stretch at the flame tip for SWQ and FF conditions. For varying $\Phi, S_{L}$ from the SWQ case (blue symbol) show an almost unique distribution along the wall normal direction, with the maximum values located at $x / \delta_{L}^{0} \approx 1$. The result implies that for all cases with different $\Phi$, the burning rate starts to decrease or the flame starts to quench towards the wall at a distance of $x \approx \delta_{L}^{0}$. The calculated quenching distances $d_{q} \approx \delta_{L}^{0}$ lay fairly within the uncertainty range of measured $d_{q}$ by Dreizler and Böhm [2] and Häber and Suntz [6] for SWQ of rod-stabilized premixed methane/air flames based on $\mathrm{OH}^{*} / \mathrm{CH}^{*}$ chemiluminescence and near-wall temperature distributions. $S_{L} / S_{L, 0}$ is larger than unity for $x / \delta_{L}^{0}>1$ due to tangential stretching of the flame with $K a_{s}>0$ and a negative
$M a$ based on $K a_{s}$ (see Fig 5 on the left too). This behavior can be confirmed also from the flame normal profiles of $\dot{r}_{\mathrm{CH}_{4}}$ shown in Fig, 2 on the right, where $\dot{r}_{\mathrm{CH}_{4}}$ at the locations 3 and 4 are larger in magnitude compared with the Cantera-solution. A further simulation has been performed for the $\Phi=1$ flame with a twice-refined grid of $\Delta=10 \mu \mathrm{m}$, which yields almost identical results for $K a$ and $S_{L}$ as given in Fig 4 (not presented here), which validates the grid resolution used in this work.
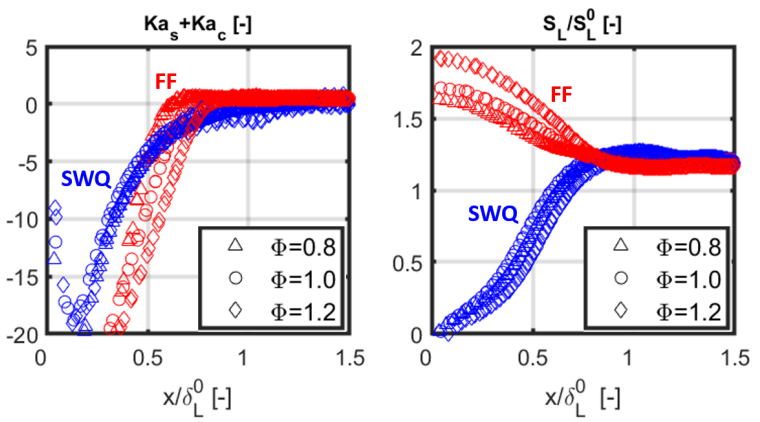

Figure 4: Comparison of $K a_{\text {tot }}$ (left) and $S_{L}$ (right) over $x$ at SWQ (blue) and FF (red) conditions.

\subsection{Markstein Numbers}

In Fig 5 on the left, $S_{L}$ increases with $K a_{s}$ both for the FF and SWQ cases, indicating a negative $M a_{s}=$ $-\frac{\mathrm{d} S_{L} / S_{L}^{0}}{\mathrm{~d} K a_{s}}$ for the tangential strain. $M a_{S, S W Q}$ is however smaller compared to $M a_{s, F F}$, which can be detected from the slopes of the curves. Regarding the effect of flame curvature or normal stretch $K a_{c}$ on $S_{L}$ shown in the center part of Fig 5, the vertical distribution of $S_{L}$ with $K a_{c}$ represents the flame branch close to the FWI zone, where $K a_{s}$ decreases towards the wall at almost constant $K a_{c} \approx 0$, as shown in Fig 3 by the blue symbols. $S_{L}$ decreases with decreasing $K a_{c}$ while approaching the cold wall (lower left branch in the center plot of Fig 5 and it increases with decreasing $K a_{c}$ for the FF case (upper left branch), yielding a reversed sign for $M a_{c}$ regarding both SWQ and FF setups. As a consequence, $M a_{t o t}$ considering the joint effect of $K a_{s}$ and $K a_{c}$ switches its sign from positive to negative too, as shown in Fig 5 on the right.

To summarize, $S_{L}$ increases with $K a_{s}$ and decreases with $K a_{c}$ in the FF case, yielding a different sign for $M a_{s, F F}$ and $M a_{c, F F}$. For the SWQ case however, $S_{L}$ is positively correlated with both $K a_{s}$ and $K a_{c}$, leading to negative values for both $M a_{s, S W Q}$ and $M a_{c, S W Q}$. The overall effect of stretch is, that $M a_{t o t, F F}$ is positive and $M a_{t o t, S W Q}$ is negative. In addition, the rates of change of $S_{L}$ with $K a_{s}, K a_{c}$ and $K a_{t o t}$ are similar in magnitude for 


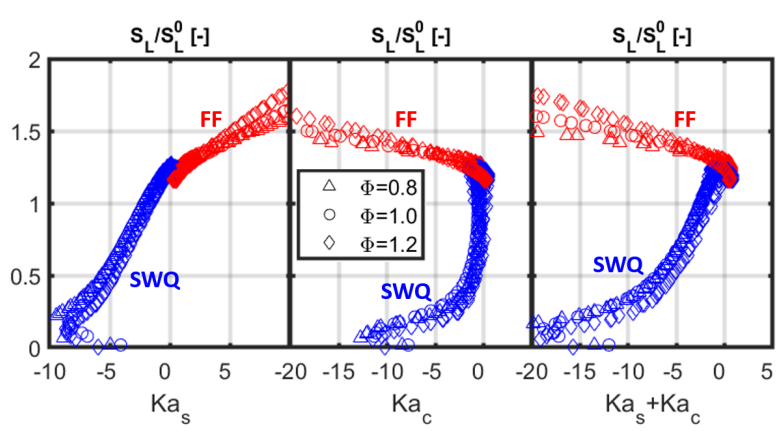

Figure 5: Correlations of flame consumption speed with flame stretch.

different $\Phi$ in the FWI zone (see Tab 2 and Fig.7 too), so that the FWI dynamics may be described with an almost constant $M a$ for different equivalence ratios. The result indicates a close connection between the quenching behaviors due to FWI and the flame dynamics, which is given by a strong decrease of $K a$ and $M a$ in the FWI zone (see Fig 3 Fig [5], resulting in a reduced consumption rate and flame speed.

In order to further validate the results for different operating conditions, DNS have been conducted for $\Phi=1.0$ with varying inlet bulk velocities $u / S_{L}^{0}=[1.1,2.0,2.9$ (base case as used in previous sections), 3.8, 5.8], which lead to different flame lengths and overall stretch rates of the flame. As shown in Fig 6 on the left, $S_{L} / S_{L}^{0}$ starts to decrease at a larger wall distance for smaller $u / S_{L}^{0}$ for the SWQ case, denoting an increase of the quenching distance with decreased bulk flow rate or flame length, respectively. $S_{L} / S_{L}^{0}$ vs. $K a_{\text {tot }}$ shown on the right of Fig 6 is shifted towards low $K a_{t o t}$ range and it decreases more slowly with decreasing $K a_{t o t}$ at higher $u$ for the SWQ flames, yielding an increase of $M a_{\text {tot }, S w Q}$. In case of FF, $S_{L}$ has its maximum value at the flame tip $\left(x / \delta_{L}^{0}=0\right)$, which increases with incoming flow rate (see the red symbols on the left of Fig 6. In this case, the flame curvature is more negative at higher $u / S_{L}^{0}$, causing an increase of $S_{L}$ due to a positive $M a_{t o t, F F}$. $M a_{t o t, F F}$ is not sensitive to $u$, as illustrated by the red symbols in Fig 6 on the right.

Table 2 lists $M a_{t o t}$ evaluated from linear curve-fitting of $S_{L} / S_{L}^{0}$ over $K a_{\text {tot }}$ for the range of $x / \delta_{L}^{0}<1$, where the data for $\Phi=0.6$ and $\Phi=1.4$ are obtained from DNS using the same numerical setups but a decreased inlet velocity to stabilize the flame. These values are plotted in Fig 7 against $\Phi$ on the left and $u / S_{L}^{0}$ on the right. It is evident that $M a_{t o t}$ reverses its sign from positive to negative while switching from the FF to the SWQ condition. $M a_{t o t, F F}$ increases monotonously with $\Phi$, whereas $M a_{t o t, S W Q}$ increases with $\Phi$ till reaching a
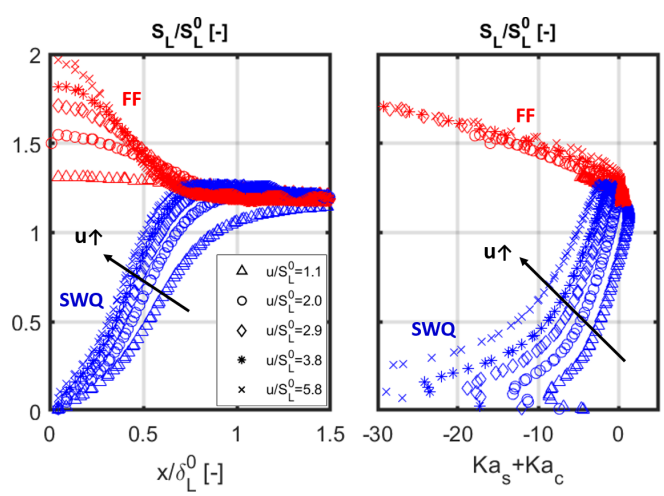

Figure 6: Effect of increased mass flow rate or overall stretch on flame consumption speed for the SWQ and FF flames.

\begin{tabular}{c|ccc}
\multicolumn{4}{c}{ Table 2: Variations of $M a_{\text {tot }}$ with $\Phi$ and $u}$. \\
$\phi[-]$ & $M a_{\text {tot }, S W Q}$ & $M a_{\text {tot }, F F}$ & $\Delta M a_{\text {tot }}$ \\
\hline 0.6 & -0.1020 & 0.0011 & 0.1031 \\
0.8 & -0.0645 & 0.0102 & 0.0748 \\
1.0 & -0.0751 & 0.0189 & 0.0941 \\
1.2 & -0.0743 & 0.0272 & 0.1015 \\
1.4 & -0.0874 & 0.0331 & 0.1206 \\
$u / S_{L}^{0}[-]$ & $M a_{t o t, S W Q}$ & $M a_{\text {tot }, F F}$ & $\Delta M a_{\text {tot }}$ \\
\hline 1.1 & -0.1022 & 0.0201 & 0.1223 \\
2.0 & -0.0946 & 0.0206 & 0.1152 \\
2.9 & -0.0751 & 0.0189 & 0.0941 \\
3.8 & -0.0620 & 0.0164 & 0.0784 \\
5.8 & -0.0461 & 0.0123 & 0.0584
\end{tabular}

peak value at $\Phi=0.8$ and it decreases again. $M a_{\text {tot }, F F}$ decreases slightly and $M a_{t o t, S W Q}$ increases with increasing flow rate or overall flame stretch, as shown on the right of Fig 7. In this case, the flame becomes more insensitive to flow stretch, yielding a decrease of the magnitudes of $\left|M a_{t o t, S W Q}\right|$ and $\left|M a_{t o t, F F}\right|$. The differences $\Delta M a_{t o t}=M a_{t o t, F F}-M a_{t o t, S W Q}$ shown in the last column of Tab2 are of the order of $O(0.1)$ for all cases, which may be considered for modeling the effect of flame quenching due to a cold wall. In order to do this, a reduction of $M a$ in Eq. (4) can be implemented by means of $\Delta M a_{t o t}$ for the FWI zone with $x / \delta_{L}^{0}<1$, which results in a decrease of the flame speed or the consumption rate.

\section{Conclusion}

Direct numerical simulations (DNS) using detailed reaction kinetics with the GRI-3.0 mechanism and mixture-averaged molecular transport have been performed to study flame dynamics of laminar premixed methane/air flames interacting with a cold wall. Different components of flame stretch and Markstein number regarding tangential, normal (due to curvature) and total stretch $K a_{s}, K a_{c}$ and $K a_{t o t}$, as well as the local flame 


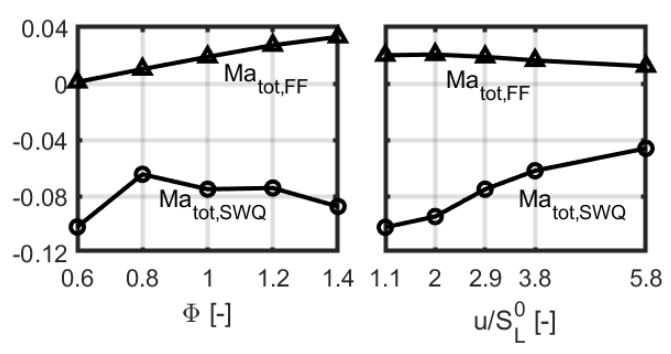

Figure 7: Comparison of $M a_{\text {tot }}$ calculated from SWQ and FF setups.

consumption speed $S_{L}$ have been evaluated based on the DNS results. The findings are summarized below:

1. FWI is dominated by negative flame stretch. The flame experiences a compression while approaching a cold wall due to a deceleration of the flow at the wall, whereas it is elongated in case of freely propagating flames (FF) without influence of a cold wall. This leads to an inversion of the sign of $K a_{s}$ from positive to negative and a decrease of $K a_{t o t}$ in the FWI zone compared with FF.

2. Signs of $M a_{c}$ and $M a_{\text {tot }}$ regarding $K a_{c}$ and $K a_{t o t}$ reverse from positive to negative in the FWI zone, leading to a decrease of $S_{L}$ with decreasing $K a_{t o t}$ while approaching a cold wall.

3. The quenching distance $d_{q}$ evaluated from $S_{L}$ has been found to be almost equal to the unstretched laminar flame thickness $d_{q} \approx \delta_{L}^{0}$, and it shows a quantitatively good agreement with measured data.

The results reveal a strong correlation between flame dynamics and the phenomena of FWI, which may open a new opportunity for modeling the effect of FWI by means of $M a$. In an attempt to do this, the flame quenching behavior due to a cold wall can be reproduced by an inversion of the sign of $M a_{t o t}$ from positive to negative within the FWI zone and applying the linear Markstein correlation for flame speed. Compared with common approaches for modeling FWI with tabulated chemistry or detailed kinetics, the way of flame dynamics may be more convenient and has the potential to reduce the computational cost significantly.

The current work has been conducted only for premixed methane/air flames, where $M a_{t o t}$ is slightly positive at FF condition. For lean-hydrogen flames however, $M a_{t o t}$ is essentially negative due to the effect of thermaldiffusive instability [1, 16]. Therefore, $M a_{\text {tot }}$ should remain its negative sign while approaching the wall in this case, which represents an opposite effect of $M a_{t o t}$ for methane/air flames obtained in the present work. Future works are therefore required to investigate an extended range of operating conditions, considering effects such as different fuels, wall temperatures and turbulent flows.

\section{Acknowledgments}

This work is supported by the Helmholtz Association of German Research Centers (HGF), within the research field Energy, Material and Resources, Topic 4 Gasification (34.14.02), and the German Research Foundation (DFG), grant number 237267381 - TRR 150.

\section{References}

\section{References}

[1] T. Poinsot, D. Veynante, Theoretical and numerical combustion, RT Edwards, Inc., 2005.

[2] A. Dreizler, B. Böhm, Advanced laser diagnostics for an improved understanding of premixed flame-wall interactions, $\mathrm{P}$ COMBUST INST 35 (1) (2015) 37-64.

[3] C. Jainski, M. Rißmann, B. Böhm, J. Janicka, A. Dreizler, Sidewall quenching of atmospheric laminar premixed flames studied by laser-based diagnostics, COMBUST FLAME 183 (2017) 271-282.

[4] A. Heinrich, S. Ganter, G. Kuenne, C. Jainski, A. Dreizler, J. Janicka, 3d numerical simulation of a laminar experimental swq burner with tabulated chemistry, FLOW TURBUL COMBUST 100 (2) (2018) 535-559.

[5] S. Ganter, A. Heinrich, T. Meier, G. Kuenne, C. Jainski, M. C. Rißmann, A. Dreizler, J. Janicka, Numerical analysis of laminar methane-air side-wall-quenching, COMBUST FLAME 186 (2017) 299-310

[6] T. Häber, R. Suntz, Effect of different wall materials and thermal-barrier coatings on the flame-wall interaction of laminar premixed methane and propane flames, INT J HEAT FLUID FL 69 (2018) 95-105.

[7] C. Strassacker, V. Bykov, U. Maas, Redim reduced modeling of quenching at a cold wall including heterogeneous wall reactions, INT J HEAT FLUID FL 69 (2018) 185-193.

[8] D. Mejia, L. Selle, R. Bazile, T. Poinsot, Wall-temperature effects on flame response to acoustic oscillations, P COMBUST INST 35 (3) (2015) 3201-3208.

[9] H. Kosaka, F. Zentgraf, A. Scholtissek, L. Bischoff, T. Häber, R. Suntz, B. Albert, C. Hasse, A. Dreizler, Wall heat fluxes and co formation/oxidation during laminar and turbulent side-wall quenching of methane and dme flames, INT J HEAT FLUID FL 70 (2018) 181-192.

[10] T. Poinsot, D. C. Haworth, G. Bruneaux, Direct simulation and modeling of flame-wall interaction for premixed turbulent combustion, COMBUST FLAME 95 (1-2) (1993) 118-132.

[11] P. Popp, M. Baum, Analysis of wall heat fluxes, reaction mechanisms, and unburnt hydrocarbons during the head-on quenching of a laminar methane flame, COMBUST FLAME 108 (3) (1997) 327-348.

[12] G. Frank, J. Zips, M. Pfitzner, Construction of libraries for non-premixed tabulated chemistry combustion models including non-adiabatic behaviours due to wall heat losses, Sonderforschungsbereich/Transregio 40 Annual Report (2014).

[13] F. Proch, A. Kempf, Modeling heat loss effects in the large eddy simulation of a model gas turbine combustor with premixed flamelet generated manifolds, P COMBUST INST 35 (3) (2015) 3337-3345.

[14] C. Strassacker, V. Bykov, U. Maas, Redim reduced modeling of flame quenching at a cold wall-the influence of detailed transport models and detailed mechanisms, COMBUST SCI TECHNOL 191 (2) (2019) 208-222. 
[15] T. C. Lieuwen, Unsteady combustor physics, Cambridge University Press, 2012

[16] F. Zhang, T. Zirwes, P. Habisreuther, H. Bockhorn, Effect of unsteady stretching on the flame local dynamics, COMBUST FLAME 175 (2017) 170-179.

[17] D. Bradley, P. Gaskell, X. Gu, Burning velocities, markstein lengths, and flame quenching for spherical methane-air flames: a computational study, COMBUST FLAME 104 (1-2) (1996) 176-198.

[18] V. P. Karpov, A. N. Lipatnikov, P. Wolanski, Finding the markstein number using the measurements of expanding spherical laminar flames, COMBUST FLAME 109 (3) (1997) 436-448.

[19] T. Tahtouh, F. Halter, C. Mounaïm-Rousselle, Measurement of laminar burning speeds and markstein lengths using a novel methodology, COMBUST FLAME 156 (9) (2009) 1735-1743.

[20] F. Halter, T. Tahtouh, C. Mounaïm-Rousselle, Nonlinear effects of stretch on the flame front propagation, COMBUST FLAME 157 (10) (2010) 1825-1832.

[21] A. Bonhomme, L. Selle, T. Poinsot, Curvature and confinement effects for flame speed measurements in laminar spherical and cylindrical flames, COMBUST FLAME 160 (7) (2013) 12081214.

[22] F. Wu, W. Liang, Z. Chen, Y. Ju, C. K. Law, Uncertainty in stretch extrapolation of laminar flame speed from expanding spherical flames, P COMBUST INST 35 (1) (2015) 663-670.

[23] T. J. Poinsot, S. Lelef, Boundary conditions for direct simulations of compressible viscous flows, J COMPUT PHYS 101 (1) (1992) 104-129.

[24] G. P. Smith, D. Golden, M. Frenklach, N. Moriarty, B. Eiteneer, M. Goldenberg, C. Bowman, R. Hanson, S. Song, W. Gardiner Jr, et al., Grimech 3.0 reaction mechanism, Sandia National Laboratory (2000).

[25] F. Zhang, H. Bonart, T. Zirwes, P. Habisreuther, H. Bockhorn, N. Zarzalis, Direct numerical simulation of chemically reacting flows with the public domain code openfoam, in: High Performance Computing in Science and Engineering'14, Springer, 2015, pp. 221-236.

[26] E. Komen, A. Shams, L. Camilo, B. Koren, Quasi-dns capabilities of openfoam for different mesh types, COMPUT FLUIDS 96 (2014) 87-104.

[27] S. Vo, O. Stein, A. Kronenburg, M. Cleary, Assessment of mixing time scales for a sparse particle method, COMBUST FLAME 179 (2017) 280-299.

[28] T. Zirwes, F. Zhang, P. Habisreuther, M. Hansinger, H. Bockhorn, M. Pfitzner, D. Trimis, Quasi-dns dataset of a piloted flame with inhomogeneous inlet conditions, FLOW TURBUL COMBUST (2019).

[29] T. Zirwes, F. Zhang, T. Häber, H. Bockhorn, Ignition of combustible mixtures by hot particles at varying relative speeds, COMBUST SCI TECHNOL 191 (1) (2019) 178-195.

[30] F. Zhang, T. Zirwes, P. Habisreuther, H. Bockhorn, D. Trimis, H. Nawroth, C. O. Paschereit, Impact of combustion modeling on the spectral response of heat release in les, COMBUST SCI TECHNOL (2018) 1-21.

[31] T. Zirwes, F. Zhang, J. A. Denev, P. Habisreuther, H. Bockhorn, Automated code generation for maximizing performance of detailed chemistry calculations in openfoam, in: High Performance Computing in Science and Engineering'17, Springer, 2018, pp. 189-204.

[32] T. Zirwes, F. Zhang, J. A. Denev, P. Habisreuther, H. Bockhorn, D. Trimis, Improved vectorization for efficient chemistry computations in openfoam for large scale combustion simulations, in: High Performance Computing in Science and Engineering'18, Springer, 2019, pp. 209-224.

[33] F. Foucher, S. Burnel, C. Mounaïm-Rousselle, M. Boukhalfa,
B. Renou, M. Trinite, Flame wall interaction: effect of stretch, EXP THERM FLUID SCI 27 (4) (2003) 431-437. 